\title{
Solar Dynamics Observatory (SDO) HGAS Induced Jitter
}

\author{
Carl Blaurock ${ }^{1}$ \\ Nightsky Systems, Inc., 3916 Lauriston Rd., Raleigh, NC 27616 \\ and \\ Kuo-Chia Liu' ${ }^{2}$ Peter Mule ${ }^{3}$ \\ NASA Goddard Space Flight Center, Greenbelt, MD 20771
}

\begin{abstract}
This paper presents the results of a comprehensive assessment of High Gain Antenna System induced jitter on the Solar Dynamics Observatory. The jitter prediction is created using a coupled model of the structural dynamics, optical response, control systems, and stepper motor actuator electromechanical dynamics. The paper gives an overview of the model components, presents the verification processes used to evaluate the models, describes validation and calibration tests and model-to-measurement comparison results, and presents the jitter analysis methodology and results.
\end{abstract}

\section{Nomenclature}

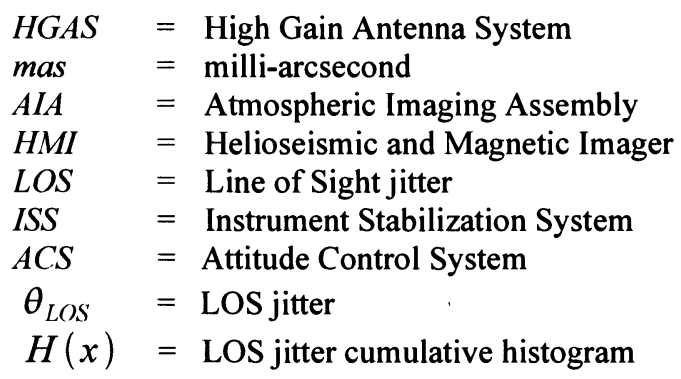

\section{Introduction}

$\mathrm{T}$ HE Solar Dynamics Observatory (SDO) is scheduled for launch in January 2009 . The main science objective of this mission is to observe the Sun and increase the understanding of the Sun's influence on Earth. There are three instruments on board the SDO for achieving its scientific goals: the Atmospheric Imaging Assembly (AIA), the Helioseismic and Magnetic Imager (HMI), and the Extreme Ultraviolet Variability Experiment (EVE). Figure 1 shows the observatory with major components annotated. Both AIA and HMI are sensitive to high frequency pointing perturbations and have sub-arcsecond level line-of-sight (LOS) jitter requirements. Disturbance sources include the reaction wheels, High Gain Antenna System (HGAS) actuators, and various mechanisms such as rotating filter wheels and shutters on the instruments. This

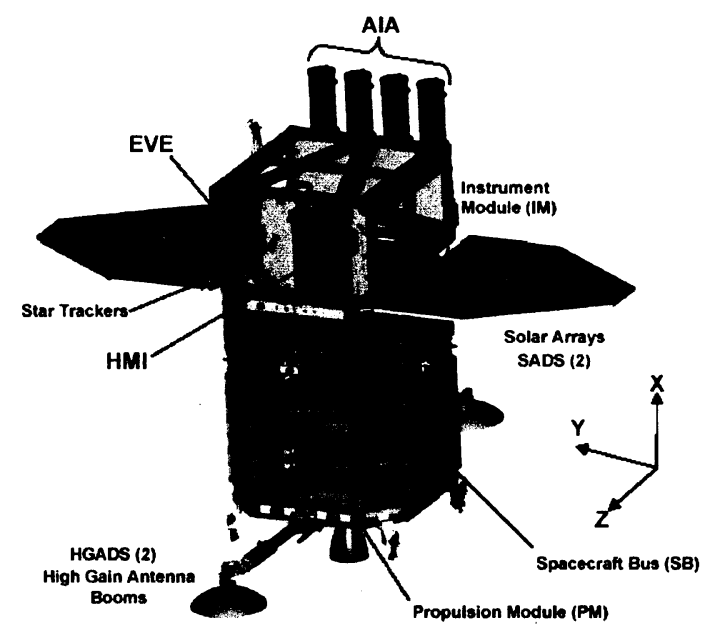

Figure 1. Solar Dynamics Observatory.

' President, 3916 Lauriston Rd., Raleigh NC 27616, and AIAA Member.

${ }^{2}$ Aerospace Engineer, Guidance and Control System Engineering Branch, Code 591, and AIAA Lifetime Member.

${ }^{3}$ Aerospace Engineer, Mechanical Systems Analysis and Simulation Branch, Code 542. 
paper describes the models and methodology used to generate the predictions of HGAS-induced instrument jitter.

The HGAS jitter predictions were developed using a complicated integrated model which will be detailed in this paper. In order to ensure the accuracy of the predictions, numerous checks were performed on each component model and on the coupled models at various stages of integration, encompassing all of the following:

- Verification: The process of determining that a computational model accurately represents the underlying mathematical model and its solution ${ }^{1}$.

- Validation: The process of determining the degree to which a model is an accurate representation of the real world from the perspective of the intended uses of the model ${ }^{2}$.

- Calibration: The process of adjusting numerical or physical modeling parameters in the computational model for the purpose of improving agreement with experimental data ${ }^{2}$.

The paper begins with an overview of the HGAS. Then the component models are presented, along with any verification, validation, and calibration activities performed by the responsible organizations. Next, the calibration and validation tests performed by the SDO Jitter team are described. Finally, the analysis predictions are presented. The paper concludes with a summary of the SDO HGAS jitter analysis, lessons learned, and recommendations for future missions.

\section{Objective and Approach}

The objective of the SDO jitter modeling activities was to analytically verify the LOS jitter requirements for HMI and AIA. The HMI jitter requirement was 140 milli-arcseconds (mas) RMS over a 0.15 second exposure. There was also a mis-registration requirement of 33.3 mas that applied to exposure-to-exposure stability over a 4 to 4.5 second cadence time between images. The AIA jitter requirement was 170 mas RMS over a 0.1 to 0.5 second exposure. The jitter requirements were sub-allocated in root-sum-square to the various disturbance sources. The allocation to the HGAS was 94 mas for HMI, and 106 mas for AIA. The AIA and HMI had data continuity budgets which allowed for occasional loss of science data. For AIA, the data continuity requirement was $96 \%$ for all sources. For HMI, sequential images are processed in aggregate, so loss of one image would lead to the loss of all images in the sequence. Therefore HMI must meet its allocation for all images. The jitter analysis was also used to verify looser requirements on spacecraft roll and antenna pointing, which are not treated in this paper.

The HGAS jitter requirements were verified by assembling models of the HGAS actuators, spacecraft structure, control systems, and instrument optics into an integrated model, the HGAS Jitter model, and simulating LOS response during HGAS operation. Model validation was performed on the component models. As will be described, some models were only partially validated, so a $100 \%$ margin on jitter was specified, leading to a target of 47 mas for HMI, and 53 mas for AIA.

\section{High Gain Antenna System Overview}

The High Gain Antenna System (HGAS) comprises two dual-axis antennae projecting from the spacecraft bus in the positive and negative $Z$ axis directions. Each antenna is driven by stepper motor actuators in azimuth and elevation. The actuators, fabricated by Spacedev, Inc., Cary, North Carolina, each consist of a 3-phase, 40-pole stepper motor providing 240 detents, with a 200:1 harmonic drive, for a final output step size of 0.0075 degrees. Each actuator step imparts a small disturbance torque into the spacecraft. The sequence of steps as the actuators move to follow the commanded profile can interact in complex ways with the ring-down from previous steps by any of the four actuators, necessitating a comprehensive jitter analysis encompassing the responses of the structure and optics, the behavior of the control systems, and the gimbal move profiles.

The HGAS control system was designed with several features to reduce induced disturbances ${ }^{4}$. First, only a single antenna is needed to meet data transmission rates, so the other antenna will be "parked" (motionless in both axes). Both antennae will be constantly emitting, so the parked position will target the beam well away from the Earth and from other spacecraft. Each of the two antennae will be parked for a portion of the year. For the remaining time, the ground station will be shielded by the spacecraft for a portion of the day for both antennae. Therefore, a "handoff" operation will be used to swap the active and parked antennae twice per day (each antenna will be active half the day). The inactive antenna will rotate in azimuth, so that the azimuth angle does not have to be driven quickly through large angles and since this motion imparts an acceptably small disturbance. The elevation axis will be fixed. This configuration is called "half-park".

In addition to the park/half-park capabilities, the HGA control algorithm incorporates a random step algorithm and a step delay. The random step algorithm adds a pseudo-random delay of zero to 200 milliseconds to each step, to ensure that the antenna does not move with a constant step rate that lines up with and excites a problematic structural response. The step delay ensures that the $+Z$ and $-Z$ actuators do not step in the same 0.2 second control

2

American Institute of Aeronautics and Astronautics 
cycle, so that actuator disturbances do not add together to double the jitter. Finally, during HGA jitter analysis it became clear that the HMI requirement could not be met $100 \%$ of the time, but that the peak jitter response decayed quickly enough that it would be possible to prevent image degradation by synchronizing the exposure and the antenna motion. An additional capability was added to enable the instruments to request the ACS to prevent actuator motion within a certain time period, by sending a do-not-step flag. The flag allows jitter response from previous steps to decay, and prevents new jitter, during the instrument exposure.

The actuators are operated in a cardinal stepping mode, so that the induced disturbances occur at the driving step rate and harmonics. Higher harmonics are of decreasing but still significant amplitude until the resonance frequency of the armature at around $60 \mathrm{~Hz}$, above which they attenuate. The disturbance harmonics vary as the step rate varies to follow a prescribed move profile, and therefore continually walk across jitter-critical structural modes. The step rates during nominal operation range between 0.25 and 1.2 pulses per second (PPS) in azimuth, and 0 to 0.5 PPS in elevation. Two strategies for the swap were examined. The first limits the elevation rates to 1 PPS during handover. The second allows the gimbals to move at 66 PPS during handover, trading degradation of science data during the swap against quicker return to nominal operation.

\section{Models and Model Verification}

The HGAS Jitter model consisted of a linear model of the closed loop spacecraft structure, comprising a linearized ACS model impinged on a linear Finite Element Model (FEM) of the structure, embedded in a nonlinear simulation of the stepper motor actuator electrodynamics. The FEM included detailed models of the AIA and HMI instruments, including all optical nodes. A linear Optical Sensitivity Matrix (OSM) was used to compute LOS at the HMI and AIA detectors as a function of motion of the optics. This section will describe the models, identify calibration parameters, and present verification processes. Calibration and validation activities performed by the responsible organization, prior to delivery of the model to the Jitter team, will also be described here.

\section{Structural Model}

The structural dynamics were assumed to be linear. The damping was assumed to be proportional (modal) with a constant value for all modes. The SDO FEM was developed to provide observatory modal information for use with advanced jitter analytical tools. The FEM represented the SDO in an on-orbit configuration: fully deployed, instrument covers open, and with $25 \%$ fuel and oxidizer mass. The FEM included the spacecraft primary structure (Propulsion Module, Spacecraft Bus, and Instrument Module [IM]), spacecraft secondary structure (High Gain Antenna System [HGAS], Solar Array System [SAS] and hardware mounting brackets), and detailed instrument models (AIA, HMI, and EVE). Instrument models were provided by Lockheed Martin and Laboratory for Atmospheric and Space Physics (LASP), and the HGA model was provided by Loral. All other spacecraft modeling was done at GSFC.

The Finite Element Model used in the SDO jitter analysis included more detail than typical of observatory models used in loads analysis. The

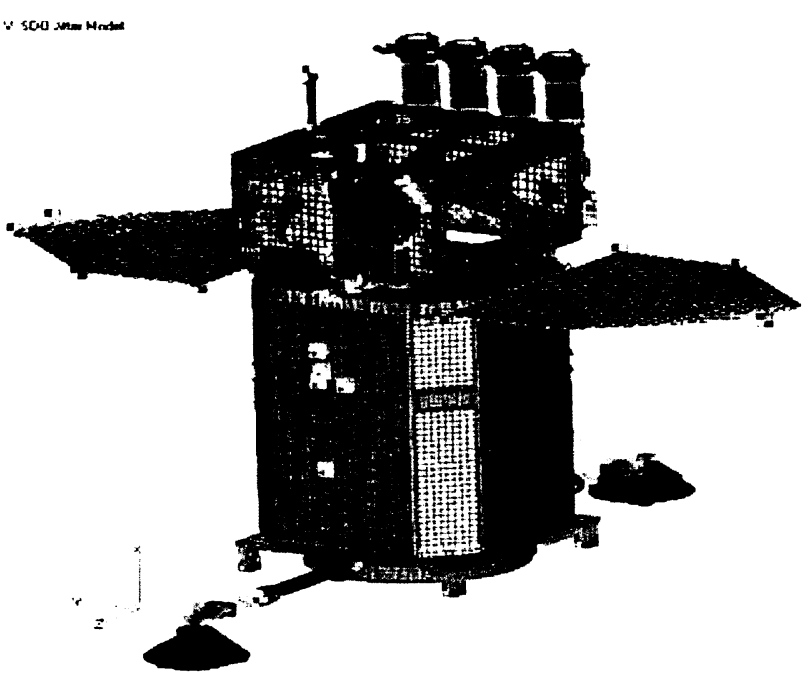
detail was necessary to more accurately predict low mass modes and higher frequency modes that have a significant effect on jitter but negligible effect on loads. An effort was made to accurately represent the mass and stiffness of the entire spacecraft and instrument structure to improve predictions for modes out to and beyond $100 \mathrm{~Hz}$. Figure 2 shows the SDO jitter model.

The final observatory jitter FEM included 461,044 nodes and 483,392 elements. To aid in the representation of the HGA actuator mechanism torsional stiffness and stepping motion, the actuator FEM includes the holding torque and gearbox stiffness along with the armature and hub inertias. Gear ratios were modeled using a Multi-Point Constraint (MPC) equation. The normal modes solution of MSC/NASTRAN was run on the unconstrained FEM, 
and mode shape and frequency data for a subset of 120 nodes was provided to the jitter group. The model was run in five different HGAS gimbal orientations to assess the effect of orientation on jitter. The analysis runs produced over 650 modes between 0 and $200 \mathrm{~Hz}$.

FEM verification activities consisted of a Unit Enforced Displacement and Rotation check, to verify that no illegal grounding was present in the model, and Free-Free Dynamics with a Stiffness Equilibrium check to verify that the model acts as a rigid body when it is unconstrained. The Stiffness Equilibrium check also verified that there are no grounding effects in the stiffness matrix.

Structural model calibration parameters included the modal damping and certain localized FEM parameters (HGAS deployment hinges and sections of the HGAS boom). A full FEM correlation was not performed due to the prohibitive cost of testing all jitter sensitive modes through $200 \mathrm{~Hz}$. The FEM correlation performed for the launch loads analysis was not completed in time for the HGAS jitter analysis, and only concentrated on modes below 50 $\mathrm{Hz}$ with significant mass participation.

\section{Optical Models}

The optical responses of the instruments (AIA, AIA Guide Telescope, and HMI) were computed using Optical Sensitivity Matrices (OSMs) provided by the instrument teams. The OSMs define the Line-of-Sight (LOS) jitter in terms of the 6-DOF motions of each of the optics:

$$
\theta_{\text {LOS }}=\sum_{\mathrm{i}=\mathrm{optic}} \sum_{\mathrm{j}=\mathrm{DOF}} C_{i j} x_{i j}
$$

where $\theta_{L O S}$ is the LOS error, $C_{i j}$ is the OSM coefficient for optic $i$ and DOF $j$, and the motions of the optics $x_{i j}$ are computed from the FEM.

The OSMs were verified by enforcing a unit displacement on the integrated structural/optical model, and ensuring that the optical response showed the correct angular LOS. Additionally, an independent ray trace was computed by the SDO Jitter team as a cross-check.

The OSMs were calibrated by measuring the focal lengths of the primary and secondary mirrors, and additionally the effective focal length of the telescope.

The OSMs were validated using interferometry tests to validate optical

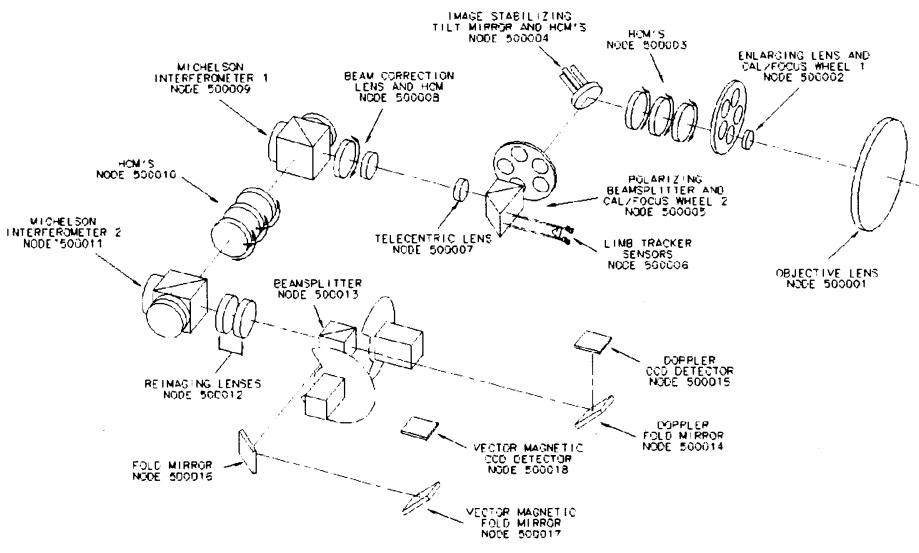
performance.

\section{Control Systems}

Three control systems influence the HGAS-induced jitter. The Attitude Control System (ACS) holds the spacecraft in a sun-pointing attitude by commanding reaction wheel torques. The Instrument Stabilization System (ISS) uses a tip/tilt mirror in each optical train to suppress jitter up to $40 \mathrm{~Hz}$. Finally, the HGAS control system commands the actuators to follow a desired profile. The models used to represent these control systems in the HGAS Jitter simulation will now be described.

The ACS control system design, the wheel torque models, and the sensor models were provided by the ACS analysis team. The attitude controller consisted of a standard proportional-integral-derivative controller and a second-order filter for suppressing flexible modes and gain stabilizing the system. It was verified by comparison to the SDO HiFi ACS simulation. Calibration activities were performed on the sensors and actuators. The ACS model was validated through system level testing at Flatsat, the SDO engineering testbed. Flatsat is a single side of all of the avionics boxes on the flight spacecraft bus, interfaced with electrical ground support equipment (EGSE) that provides simulation of the various ACS/Propulsion sensors and actuators. The EGSE also provides the command and telemetry interfaces.

The ISS controllers were designed and constructed by engineers at the Lockheed Martin Solar and Astrophysics Laboratory (LMSAL). Each AIA science telescope is accompanied by a guide telescope that uses limb sensors to provide pointing jitter signals to the ISS for image motion correction. HMI also has limb sensors to feed back error 


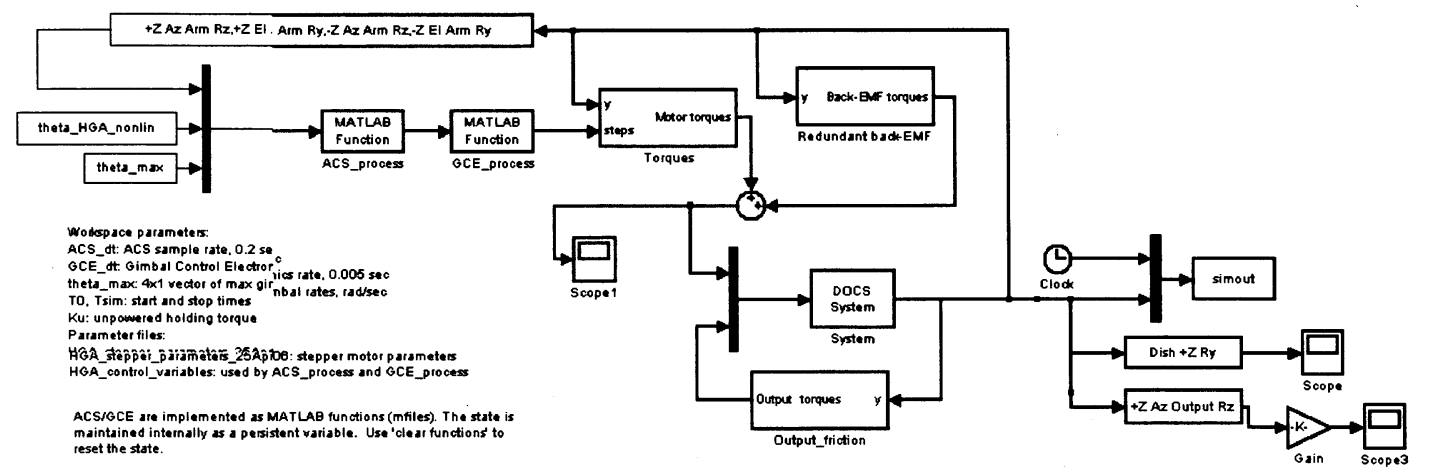

Figure 4. HGAS Jitter model.

signals to its ISS which drives a tip/tilt mirror to reduce image motion. ISS disturbance rejection was modeled with a high-pass filter. The models were verified against the Mission Requirements Document requirement, and validated by measuring achieved motion suppression. Note that when the test data showed better performance than the requirement, the requirement was used in the analysis for additional conservatism.

The HGAS control system was modeled with two MATLAB function blocks. One block, running at the $5 \mathrm{~Hz}$ ACS sample rate, computes the commanded step times based on the gimbal trajectory. The second block, running at the Gimbal Control Electronics $200 \mathrm{~Hz}$ sample rate, commands the steps to each actuator. The HGAS control system was verified against the HiFi ACS simulation.

\section{HGAS Actuator Model}

The HGAS actuator model was developed from a proprietary model of the stepper motor actuator provided by the actuator vendor. The model included powered and unpowered holding torques, back-EMF, armature friction, and gearbox friction. The same model was used for verifying torque margin for the SDO actuators, so were subject to extensive validation by the vendor. Calibration parameters include friction, detent torque, and harmonic drive stiffness and effective inertia.

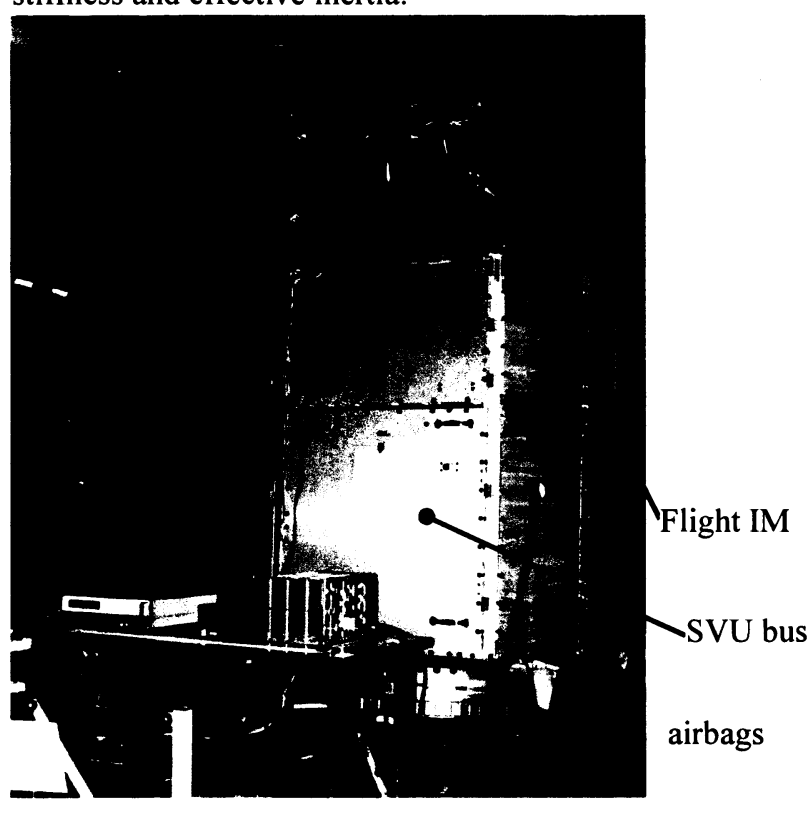

Figure 5. SVU damping characterization test.

\section{Redundant Winding Bleed Resistor Model}

The actuator includes a redundant set of windings as a backup to the primary windings. They are not used by SDO, and are shorted by a bleed resistor to prevent charge buildup. Initially, a 10k-ohm bleed resistor was used. This value resulted in a very low winding current, and thus a low back-EMF induced torque that did not affect armature motion. When the initial HGAS jitter predictions were found to be high, an investigation was conducted into the effects of using a lower bleed resistor to increase the backEMF torque and thus increase armature damping. A value of $10 \mathrm{ohms}$ was found to decrease jitter by about $10 \%$ on average, and to have acceptably minor effect on torque margin. The redundant winding and bleed resistor were represented by a model derived from the actuator model.

\section{HGAS Jitter Model}

The HGAS Jitter model was realized in MATLAB $®$ Simulink $\circledast$ (Figure 4). The model included the structural dynamics as a first-order state 
space model, as well as nonlinear elements describing the powered and unpowered holding torques (replicated four times for the four actuators), the HGAS control system blocks, and the bleed resistor model.

\section{Model Calibration}

The damping model and actuator model were calibrated by the Jitter team. This section describes the hardware, Ground Support Equipment (GSE), measurements, data processing, and results for each test.

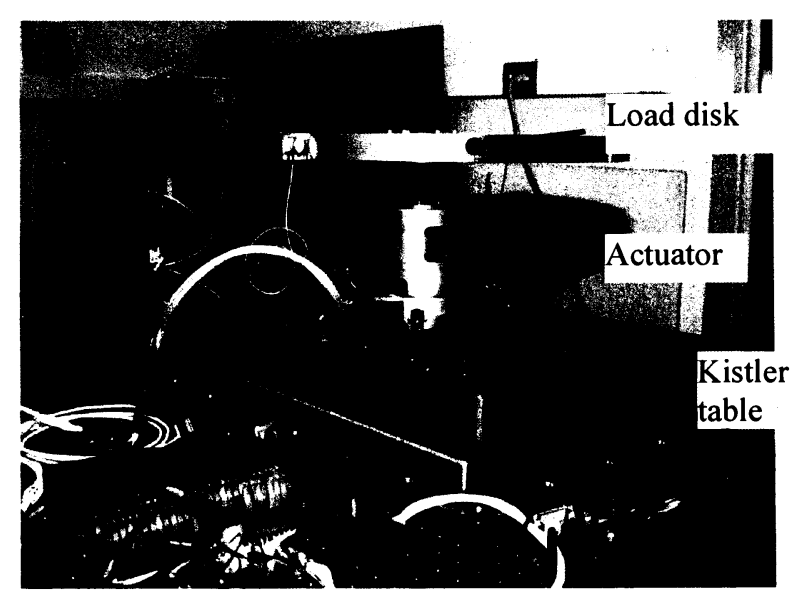

Figure 7. Actuator characterization test.
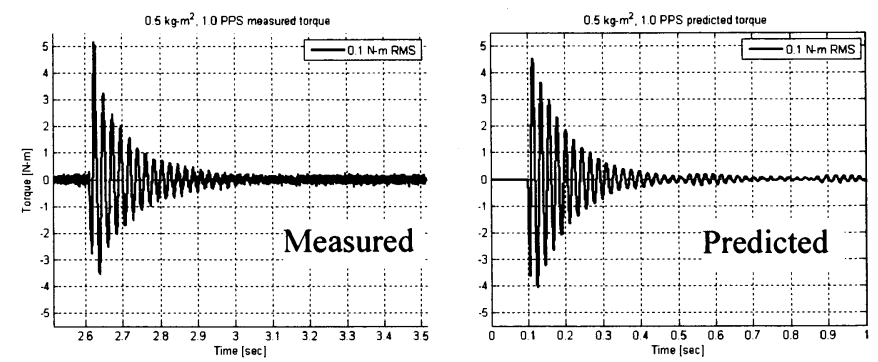

Figure 8. Predicted and measured disturbance torques.

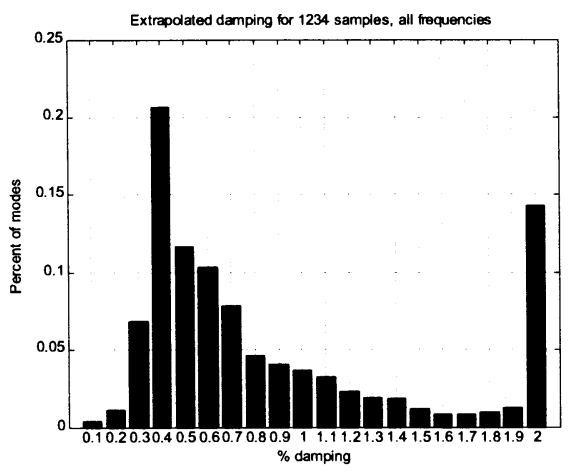

Figure 6. Damping histogram.

\section{Damping Model}

The damping model was calibrated using the Strength Verification Unit (SVU) bus. The SVU, with flight IM, was mounted on airbags to provide a flight-like boundary condition. A shaker with load-cell-equipped stinger was used to introduce forces at 5 locations around the structure, at three forcing amplitudes. 19 accelerometer channels were acquired up to 200 Hz. Frequency Response Functions (FRFs) were computed and fit using system identification tools to produce models of the spacecraft dynamics. The system damping ratios were extracted and extrapolated down to flight response amplitudes by matching RMS accelerations to predictions of flight levels. The test setup is shown in Figure 5. Figure 6 is a histogram of damping ratios for all identified modes. The value of $0.3 \%$ critical damping bounded $97 \%$ of the identified modes and was used in the jitter analyses.

\section{Actuator Model}

The HGAS actuator model was calibrated in two separate tests. The parameters dominating mechanical forcing were calibrated by mounting

a representative load to the actuator output, commanding steps at several step rates, and measuring the induced disturbance. Figure 7 shows the test setup, including the actuator, Kistler table for measurement of 6-axis forcing, and load disk. Three rigid load disks with inertias of $0.5,1.0$, and $2.0 \mathrm{~kg}-\mathrm{m}^{2}$ were fabricated, enveloping the elevation actuator load of $1.0 \mathrm{~kg}-\mathrm{m}^{2}$ and the azimuth actuator load ranging from 0.5 to $1.6 \mathrm{~kg}-\mathrm{m}^{2}$ depending on elevation angle. In addition to validating the actuator disturbance forcing prediction, the test also allowed calibration of the actuator output inertia, stiffness, and damping characteristics. After tuning the gearbox stiffness and damping, excellent agreement was found between the predicted and measured forcing (Figure 8).

The motor electrodynamic parameters were calibrated using measurements of redundant winding voltage during actuator motion. Figure 9 plots the voltages for the 3 motor phases, as
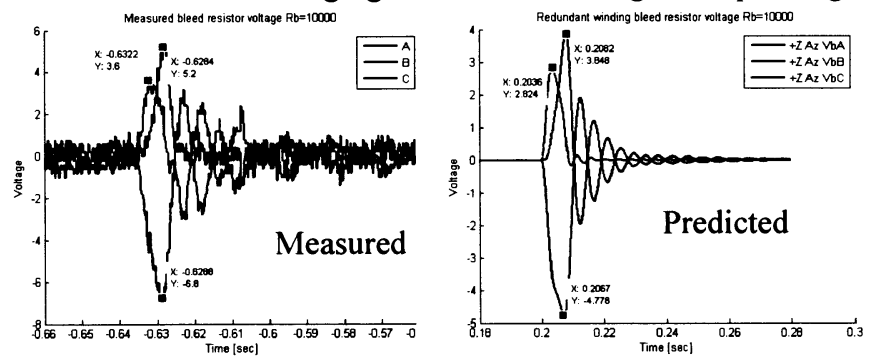

Figure 9. Predicted and measured winding voltages. 


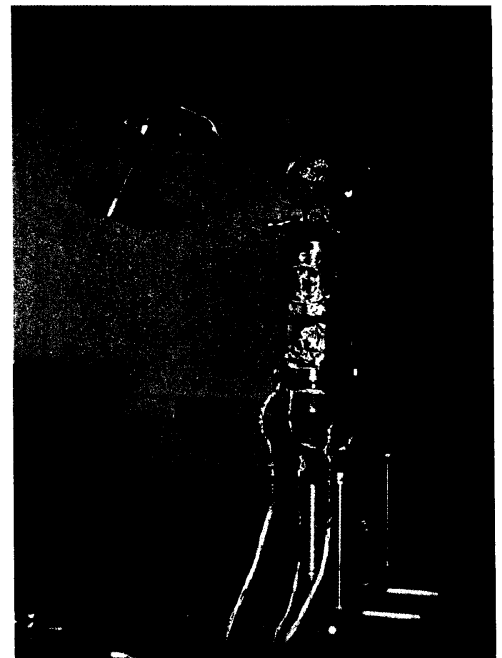

Figure 10. HGAS boom test setup measured and as predicted by the model. The waveform is captured very accurately, but the amplitude is under-predicted. This has been shown to arise from the assumption in the model that the motor constant is sinusoidal in armature angle.

\section{HGAS Boom Tap Test}

The HGAS model was calibrated using tap test data from a flight boom. The test hardware consisted of a flight boom outboard of, and including, the azimuth bracket. The boom was cantilevered to a Kistler table, instrumented with accelerometers on the boom and the dish, and tapped using an instrumented hammer. The test was repeated with the flight antenna and with an antenna mass simulator to isolate boom dynamics. Figure 10 shows the test setup with the mass simulator. Frequency response functions were used to tune a detailed shell model of the azimuth bracket, which was then used to extract a GENEL generalized element representing the 6 DOF stiffness of the bracket for use in the FEM. The resulting model match will be shown in Section VI. The deployment hinge model was also updated using static stiffness test results.

\section{Model Validation}

The calibration activities provided validation data for the actuator model. Additional validation tests were performed on the HGAS boom, and on the SVU bus.

\section{HGAS Antenna Jitter Test}

The HGAS disturbance model was validated by operating the HGAS and measuring the forces introduced on the spacecraft. The flight boom and antenna were mounted on a Kistler table, in the same test setup shown in Figure 10, and operated at several step rates in both axes. A corresponding model was created by extracting the HGAS model from the FEM, coupling it to a model of the Kistler table, and embedding the resulting Normal Modes model into the HGAS Jitter simulation. Figure 11 plots the power spectral densities (PSDs) of the forces as measured and as predicted by the model. The lower plots are the PSDs, and the upper plots are curves of cumulative RMS. The frequency characteristics in the PSD are captured very well. The model over-predicts the RMS force, since it uses a single damping ratio for all modes, matched to the lowest damping ratio in the data. Note that there is significant
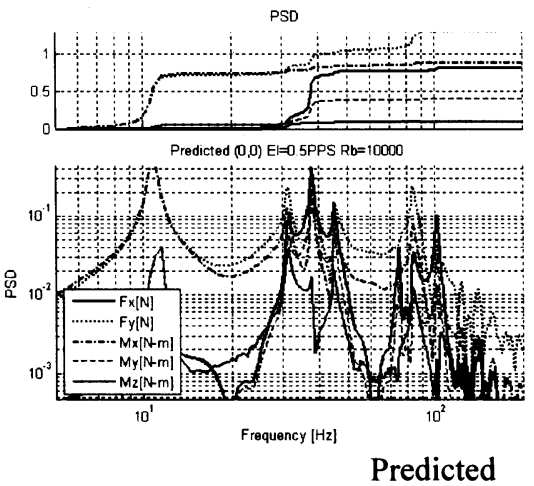
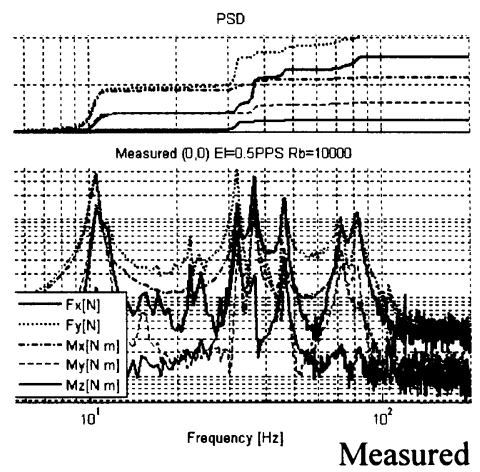

Figure 11. Predicted and measured disturbance torque.

energy content up to $100 \mathrm{~Hz}$.

\section{Reaction Wheel Jitter Model Validation}

The structural model was partially validated through a reaction wheel jitter test. Briefly, the test involved mounting an engineering development bus, flight IM, telescope simulators (a dynamic simulator for one AIA telescope, mass simulators for the other 3 AIA telescopes and the HMI telescope) on airbags, mounting a flight reaction wheel, and running at various speeds. Acceleration spectra were captured from various locations and compared to predictions, and demonstrated that the predicted jitter was a close over-bound to the measured jitter. The same structural model was used for reaction wheel and HGAS jitter prediction, so the test also validated the elements of the HGAS jitter model that were common to the RWA, including the bus, IM, and instruments. A more 
comprehensive discussion of the test procedure and results can be found in Ref. 2 .
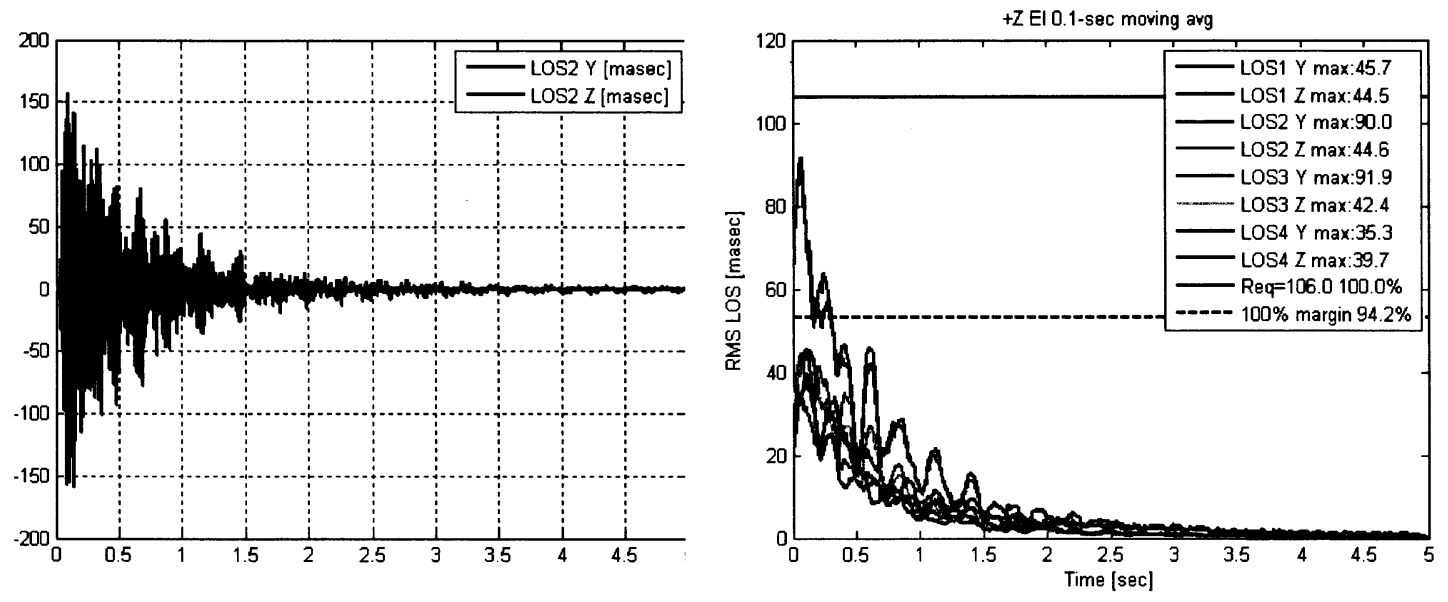

Figure 12. Simulated AIA LOS for instrument 2, and 0.1 second moving average RMS for all instruments, for a single step of the $+Z$ elevation actuator.

\section{Simulation Results}

The HGAS Jitter model was exercised using a variety of move profiles, for each of the six model configurations. A typical jitter result for a single step of the worst jitter-inducing actuator, for the $+Z$ elevation axis, is shown in Figure 12. The left plot shows the time response of AIA telescope 2, in $\mathrm{X}$ and $\mathrm{Y}$, including ISS suppression. The right hand plot shows the corresponding moving average jitter, over the shortest exposure time of 0.1 second, for all four telescopes in $\mathrm{X}$ and $\mathrm{Y}$. The peak response is 91.9 mas, seen in the $\mathrm{Y}$ axis for telescope 3. Telescope 2 is nearly the same magnitude. The peak response is below the 106 mas allocation (shown by the red line), but well above the desired $100 \%$ margin (indicated by the dashed black line). The response settles to below the target in about 0.3 seconds. Therefore there is still significant response after the 0.2 second ACS cycle time, which will interact with the response induced by the next actuator step. On average the two responses will constructively interfere half the time. Thus, as will be seen, the peak jitter during a move can be much higher than the peak single-step jitter.

Multiple simulation runs were conducted, using representative sections of the four different move profiles, and using each of the six model configurations. The peak jitter during a move profile, with multiple actuators operating, was found to be 181 mas for the worst AIA, and 160 mas for HMI. These jitter levels exceeded the entire jitter budget. No attempts at mitigation were found to be effective and achievable in terms of budget and schedule. Therefore, attention turned to quantifying the fraction of time that these jitter levels were present, with the intent of either using the data efficiency budget to accept degradation of a certain percentage of images, or alternately of determining whether there was enough time between jitter peaks to allow for exposure between actuator steps by synchronizing the instruments and the ACS.

\section{Jitter Histograms}

The jitter simulation results were processed into cumulative histograms that define the percentage of a given interval for which the jitter is less than or equal to a specified value. The histogram $H(x)$ is the value of LOS in mas that the moving average jitter is equal to or below for $x$ percent of the interval. The histograms for each operating condition ( $+Z$ track, $-Z$ track, handover, and fast handover) are computed by sorting the moving-average jitter amplitude from the time simulations for one day's operation, and separating them into bins. The histograms for the different operating conditions are then summed, weighted by the number of days spent in each operating condition, to generate the histogram for an entire year of operation,

$$
H_{1-\text { year }}^{1}=\frac{120 d}{365 d} H_{+Z \text { track }}^{-1}+\frac{161 d}{365 d} H_{\text {handoff }}^{1}+\frac{79 d}{365 d} H_{-Z_{\text {track }}}^{1}
$$

\section{8}

American Institute of Aeronautics and Astronautics 

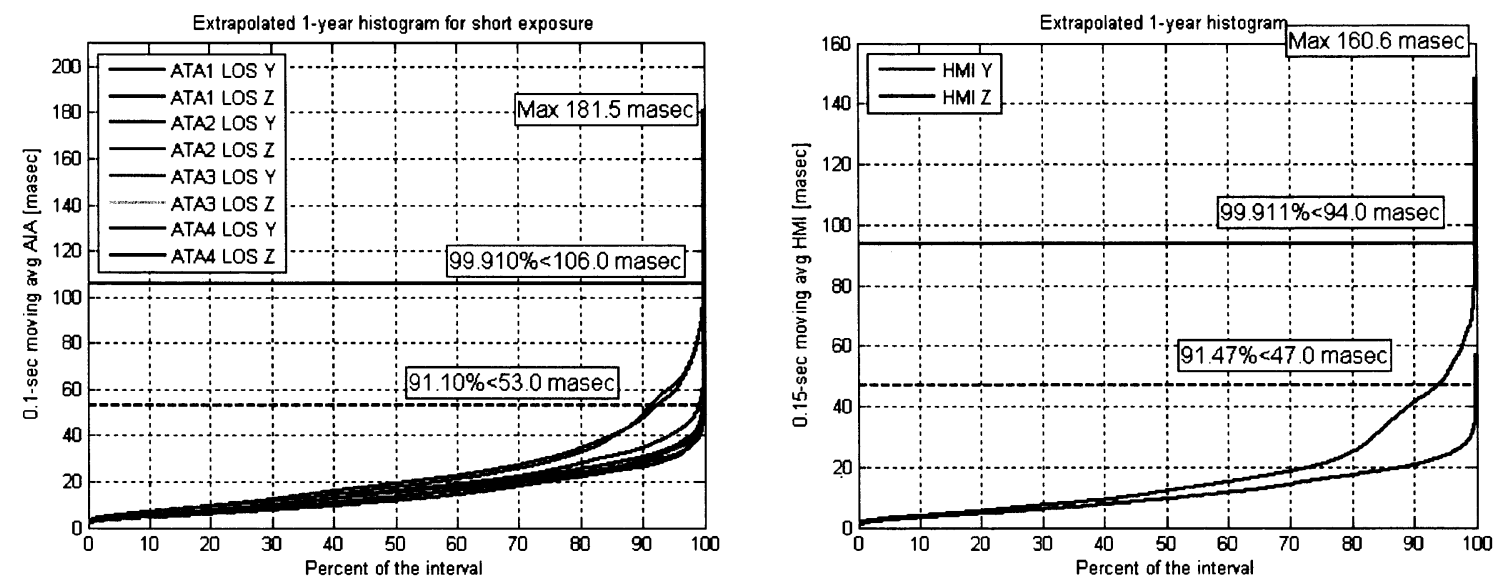

Figure 13. Jitter histograms at shortest exposure time for AIA and HMI.

The results for the AIA and HMI, for the shortest exposure time for each instrument, are plotted in Figure 13. The left hand plot shows the cumulative histogram for the four AIA telescopes, in each of two axes. The peak jitter is 181.5 mas in the worst axes (telescopes 2 and 3, in the $\mathrm{Y}$ axis). However, the jitter is below the 106 mas allocation for $99.91 \%$ of the year, and below the $100 \%$ margin level for $91.1 \%$ of the year. If all of the efficiency budget is allocated to jitter, the corresponding peak AIA jitter is 63 mas. This provides a margin of $68 \%$ on the allocation. The right hand plot shows the cumulative histogram for the HMI telescope, in 2 axes. The peak jitter is 160.6 mas. The jitter is below the 94 mas allocation for $99.911 \%$ of the time. This would still cause loss of an image sequence. However, the allocation is exceeded for only a small percentage of the time, so that it is possible to synchronize the HGA steps with HMI exposures, so that no steps are taken during HMI imaging. As a result of this finding, the do-not-step flag discussed in Section II was implemented.

\section{Conclusion}

The paper has presented the predictions of HGAS-induced jitter for the Solar Dynamics Observatory. The paper described the SDO instruments, the jitter requirements, and the details of the HGAS actuators. The models used to predict jitter were described, along with verification, validation, and calibration activities. Then simulation results were shown, demonstrating that predicted jitter exceeds the allocation. Finally, the histogram approach to computing percentage of time within the jitter allocation was presented. The results indicate that the AIAs will meet their allocation with $68 \%$ margin, assuming that all of the efficiency budget can be allocated to jitter. The HMI telescope will also meet its allocation if the HGAS steps are synchronized to avoid exciting jitter during HMI exposure.

The SDO analysis effort supports the following statements and conclusions. Jitter analysis must be performed early in the program to identify problems when design changes can be made with minimal impact. Substantial margin must be maintained since models will be immature and will not be calibrated. The FEM must be developed with jitter analysis in mind, so as to have a sufficient mesh density to maintain prediction accuracy through the frequency range where there is disturbance energy content. High modal content can create significant amplification when modes stack, beyond a typical $Q=500$ assumption from a back of the envelope calculation. SDO exhibited amplification of $10^{3}-10^{4}$ above the rigid body response in the range from 40 to $70 \mathrm{~Hz}$. The analysis models should be calibrated as much as possible, at the component, subsystem, and system level. The system should be designed to enable $1 \mathrm{G}$ testing as far as possible, with minimal use of off-loaders. SDO test data also suggest that a modal damping value of $0.3 \%$ of critical is a conservative number for jitter analysis, for a room-temperature frame and honeycomb panel spacecraft.

Specifically with respect to the HGAS actuators, the following statements can be made. First, the requirement for $1 \mathrm{G}$ testability implies non-zero detent torque. This is preferable because it enables model calibration, even though it increases the HGAS-induced jitter. In addition, jitter mitigation techniques should be examined early in the design process, including using mass balancers to center the load CG on the center of rotation, microstepping, and control system capabilities such as random and stagger stepping. Note that microstepping requires constant actuation of 
multiple actuator windings, and therefore has power and thermal impacts that must be accommodated.

\section{Acknowledgments}

The authors would like to acknowledge significant efforts from numerous individuals, including Tom Kenney (SDO GNC lead) and Wendy Morgenstern (SDO ACS lead) for coordinating tests and modeling activities; Monica Zuray, Brian Ross, Kirkland Brown, and Javier Lecha for test activities; Kristin Bourkland for HGAS algorithm assistance; David Baran, Joseph Shepis, and Richard Barklay for assistance with actuator modeling and calibration; and Joseph Hashmall and Laurie Mann for providing gimbal trajectories.

\section{References}

${ }^{1}$ ASME Guide for Verification and Validation in Computational Solid Mechanics, March 29, 2006 Draft.

${ }^{2}$ Guide for the Verification and Validation of Computational Fluid Dynamics Simulations, American Institute of Aeronautics and Astronautics, 1998, Reston, Va. AIAA G-077-1998.

${ }^{3}$ Liu, K.-C., Maghami, P., and Blaurock, C., "Reaction Wheel Disturbance Modeling, Jitter Analysis, and Validation Tests for Solar Dynamics Observatory," AIAA Guidance, Navigation, and Control Conference, AIAA, Washington, DC, 2008, to be published.

${ }^{4}$ Bourkland, K. L., Liu, K.-C., Blaurock, C., "A Jitter-Mitigating High Gain Antenna Pointing Algorithm for the Solar Dynamics Observatory", GSFC $20^{\text {th }}$ International Symposium on Space Flight Dynamics (ISSFD), 2007. 